\title{
Medindo o Impacto da Promoção do Aleitamento Materno em Serviços de Atenção Primária à Saúde em Pelotas, Rio Grande do Sul, Brasil
}

\author{
Measuring the Impact of Promoting Breastfeeding in Primary Health \\ Care Services in Pelotas, Rio Grande do Sul, Brazil
}

\author{
Marilda B. Neutzling ${ }^{1}$; Maria de Fátima Vieira ${ }^{1}$; Juraci A. César ${ }^{2}$ \\ Denise P. Gigante ${ }^{1}$; Eliana B. Martins ${ }^{1}$; Luiz A. Facchini ${ }^{2}$
}

\begin{abstract}
NEUTZLING, M. B.; VIEIRA, M. F.; CÉSAR, J. A.; GIGANTE, D. P.; MARTINS, E. B. \& FACCHINI, L. A. Measuring the Impact of Promoting Breastfeeding in Primary Health Care Services in Pelotas, Rio Grande do Sul, Brazil. Cad. Saúde Públ., Rio de Janeiro, 9 (2): 149154, Apr/Jun, 1993.
\end{abstract}

All mothers with children enrolled in the Program for Child Growth and Development at primary care units belonging to the Federal University of Pelotas (UFPel) in the state of Rio Grande do Sul, Brazil, were interviewed with a standardized questionnaire. This research aimed to assess the impact of the "Groups of Expecting Mothers" in the promotion of breastfeeding. The family income of almost half of the 347 children studied was two times the minimum wage. About 1/4 of the children's mothers had spent less than four years in school, and these were the mothers who attended the Groups most frequently. Most of the mothers received prenatal care and nearly half of then participated in the Groups. In contrast, 1/3 of the children were weaned at the age of three months and almost $80 \%$ received tea in the early months of life. The results show that the Groups of Expecting Mothers suffer serious limitations in promoting breastfeeding and in postponing the introduction of foods other than breast milk in the children's diet. Data obtained in this study are intended to help strengthen action under current programs and to show that with minimum resources and a rather simple methodology it is possible to assess the quality of health services available to the population.

Key words: Breastfeeding; Child Health; Pregnancy; Community Health

\section{INTRODUÇÃO}

Durante vários séculos, a amamentação ao seio foi a única forma de alimentação dos seres humanos nos primeiros meses de vida (Jelliffe $\&$ Jelliffe, 1978). Este processo natural e eficiente sofre a influência de vários fatores como a industrialização acelerada, incentivando o consumo de leites e fórmulas lácteas; a incor-

\footnotetext{
1 Departamento de Nutrição da Faculdade de Nutrição da Universidade Federal de Pelotas, Campus Universitário, Pelotas, RS, 96010-900, Brasil.

${ }^{2}$ Departamento de Medicina Social da Faculdade de Medicina da Universidade Federal de Pelotas, Caixa Postal 464, Pelotas, RS, 96010-900, Brasil.
}

poração da mulher ao mercado de trabalho; a pouca valorização do aleitamento natural pelos profissionais de saúde, entre outros (Manciax, 1982).

Estas mudanças provocaram alterações desastrosas no perfil de morbi-mortalidade infantil, principalmente nos países não-desenvolvidos (Jelliffe et al., 1978). Tais ocorrências fizeram com que alguns organismos internacionais, entre eles o Fundo das Nações Unidas para a Infância (Unicef) e International Baby Food Action Network (IBFAM), empreendessem esforços significativos para que esta prática fosse retomada (Boletim AAPH, 1990).

Entretanto, foi nos países industrializados que se observou um retorno progressivo ao aleita- 
mento materno, a partir das classes de melhor nível sócio-econômico (Manciax, 1982).

No Brasil, essa tendência de retorno à amamentação ainda não pôde ser observada devido ao pequeno número de estudos e à ausência de avaliação de programas a nível nacional (Monteiro, 1988). Apesar disso, a maioria dos serviços de saúde no país, principalmente a nível de rede básica, tem como uma de suas prioridades incentivar o aleitamento natural (Grant, 1992).

O incentivo ao aleitamento materno é uma prática constante nos serviços de atenção primária à saúde (APS) em Pelotas, RS. Nestes serviços foram criados os chamados "grupos de gestantes", onde, periodicamente, sob a coordenação de um médico, mulheres grávidas reuniam-se para discutir problemas relacionados à gestação e, principalmente, incentivar o aleitamento natural pelo menos até os seis meses de idade.

Entretanto, em estudo realizado em Pelotas, observou-se que a duração mediana a amamentação era pouco superior a três meses e que as mulheres pertencentes às famílias de menor poder aquisitivo amamentavam seus filhos por um período ainda menor (Victora et al., 1982).

Baseando-se nestes achados, na ausência de avaliações periódicas dos serviços de APS, nas características das populações atendidas, no tempo destinado pela equipe de saúde para incentivar o aleitamento natural, entre outros, resolveu-se fazer esta avaliação.

Assim, buscou-se responder algumas questões relativas ao assunto, como, por exemplo, a influência dos grupos de gestantes no padrão de amamentação, bem como identificar fatores ligados ao desmame precoce e à duração mediana do aleitamento natural em crianças matriculadas nos programas de puericultura das unidades sanitárias (US) da Universidade Federal de Pelotas (UFPel).

\section{METODOLOGIA}

Apesar da existência de cerca de 50 US no município de Pelotas, o presente estudo foi realizado somente naqueles locais onde existiam programas de incentivo ao aleitamento e onde a Faculdade de Nutrição desenvolvia atividades de ensino. Por estes motivos, os dados desta pesquisa dizem respeito apenas às sete US pertencentes e gerenciadas pela UFPel. Estas unidades eram compostas basicamente por médicos, nutricionistas, assistentes sociais, atendentes de enfermagem e alunos estagiários de diversos cursos.

Nestas sete unidades sanitárias foram entrevistadas todas as mães de crianças menores de um ano inscritas no programa de puericultura que visitaram estes locais durante o mês de setembro de 1990. De um total de 700 crianças inscritas com idade entre 0 e 12 meses, foram obtidas informações sobre 347 , o que revela uma cobertura pelo programa de aproximadamente $50 \%$ naquela época.

A coleta de dados foi realizada por estagiários, acadêmicos do curso de Nutrição, adequadamente treinados através de um estudo piloto e sistematicamente supervisionados por uma professora $(\mathrm{MN})$ responsável pelo estágio e por um médico epidemiologista (LF) do Departamento de Medicina Social da UFPel.

Para a coleta de dados foi utilizado um questionário padronizado que buscava informações sobre as seguintes variáveis: maternas (escolaridade, número de filhos, período pré e perinatal e utilização de anticoncepcional oral); infantis (idade, sexo, peso ao nascer, hospitalizações, amamentação, dieta e percentil de peso no momento da entrevista); e sócio-econômicas (renda familiar e número de pessoas no domicílio). Os questionários eram codificados e revisados pelos supervisores.

Foram utilizados os testes do qui-quadrado para a comparação de duas proporções. A amamentação foi calculada através da análise de sobrevivência, que permite estimar em cada intervalo - no caso, a cada mês - a proporção de crianças que ainda estão sendo amamentadas (Kirkwood, 1988). A mediana de duração da amamentação referida indica a idade na qual metade das crianças já foi desmamada (Barros \& Victora, 1991).

\section{RESULTADOS}

A maioria das 347 crianças estudadas pertencia ao sexo feminino (52\%) e apresentava idade inferior a cinco meses (58\%) (Tabela 1).

Metade das crianças estudadas era provenien- 
te de famílias com renda inferior a dois salários mínimos mensais (SM); um quarto de suas mães apresentavam escolaridade inferior a quatro anos (Tabela 2).

TABELA 1. Distribuição das Crianças de Acordo com Algumas Características Demográficas. Unidades Sanitárias da UFPel, Pelotas, RS, 1990

\begin{tabular}{lcc}
\hline \hline Características & Percentuais & $\mathrm{N}$ \\
\hline Sexo & & \\
Masculino & $48 \%$ & 168 \\
Feminino & $52 \%$ & 179 \\
& & \\
Grupos de idade & & \\
(6 meses) & $31 \%$ & 108 \\
0 a 2,9 & $27 \%$ & 93 \\
3 a 5,9 & $22 \%$ & 76 \\
6 a 8,9 & $20 \%$ & 70 \\
9 a 11,9 & & \\
\hline Total & $100 \%$ & 347 \\
\hline \hline
\end{tabular}

TABELA 2. Distribuição das Crianças Conforme Variáveis Sócio-Econômicas. Unidades Sanitárias da UFPel, Pelotas, RS, 1990

\begin{tabular}{lcc}
\hline \hline Características & Percentuais & $\mathrm{N}$ \\
\hline Anos de escolaridade & & \\
Nenhum & $6 \%$ & 20 \\
1 a 3 & $20 \%$ & 70 \\
4 a 7 & $52 \%$ & 182 \\
8 ou mais & $22 \%$ & 75 \\
& & \\
Renda familiar mensal & & \\
em salários mínimos & & \\
$<1$ & $21 \%$ & 67 \\
1 a 1,9 & $28 \%$ & 90 \\
2 a 2,9 & $24 \%$ & 76 \\
$\geq 3$ & $26 \%$ & 84 \\
\hline Total & & \\
\hline \hline
\end{tabular}

Praticamente todas as mães realizaram pelo menos uma consulta de pré-natal, sendo unidades sanitárias (48\%) e os ambulatórios hospitalares $(26 \%)$ os locais mais procurados (Tabela $3)$.

Das mães que fizeram uma ou mais consultas de pré-natal, somente $40 \%$ participaram dos grupos de gestantes; destas $90 \%$ lembravam de algum assunto discutido nas reuniões de grupo, principalmente assuntos relacionados ao parto (30\%) e a amamentação (26\%). Apenas $10 \%$ (14) delas receberam visita de um integrante da equipe de saúde no período pós-parto (Tabela 4).

TABELA 3. Distribuição das Crianças de Acordo com a Utilização dos Serviços de PréNatal e Participação nos Grupos de Gestantes. Unidades Sanitárias da UFPel, Pelotas, RS, 1990

\begin{tabular}{lcc}
\hline \hline Características & Percentuais & $\mathrm{N}$ \\
\hline Consulta de pré-natal & & \\
Não fez & $4 \%$ & 14 \\
1 ou mais & $96 \%$ & 333 \\
& & \\
Local & $48 \%$ & 167 \\
Unidade sanitária & $26 \%$ & 90 \\
Ambulatório hospitalar & $10 \%$ & 36 \\
Policlínicas & $12 \%$ & 40 \\
Outros & $4 \%$ & 14 \\
Não fez & & \\
& $40 \%$ & 137 \\
Participaram dos grupos & & \\
\hline Total & $100 \%$ & 347 \\
\hline \hline
\end{tabular}

TABELA 4. Distribuição das Crianças Conforme os Assuntos Discutidos nos Grupos de Gestantes e Visita Domiciliar. Unidades Sanitárias da UFPel, Pelotas, RS, 1990

\begin{tabular}{lcc}
\hline \hline Características & Percentuais & $\mathrm{N}$ \\
\hline $\begin{array}{l}\text { Lembram de algum } \\
\text { assunto discutido }\end{array}$ & $89 \%$ & 122 \\
& & \\
Assuntos mais lembrados & & \\
Parto & $30 \%$ & 41 \\
Amamentação & $26 \%$ & 36 \\
Outros & $44 \%$ & 60 \\
& & \\
Receberam visita & & \\
domiciliar no pós-parto & $10 \%$ & 14 \\
\hline Total & $100 \%$ & 137 \\
\hline \hline
\end{tabular}


A Figura 1 mostra que mães de menor escolaridade participaram mais freqüentemente dos grupos de gestantes.

Na Figura 2 é possível verificar que, independentemente do peso ao nascer, a maioria das crianças foi desmamada entre zero e dois meses de idade.

Oitenta por cento das crianças começaram a receber chás nos primeiros meses de vida, enquanto que a introdução de comida de sal ocorreu entre o terceiro e o quinto mês (Figura $3)$.

FIGURA 1. Distribuição das Mães de Acordo com a Escolaridade e Participação nos Grupos de Gestantes

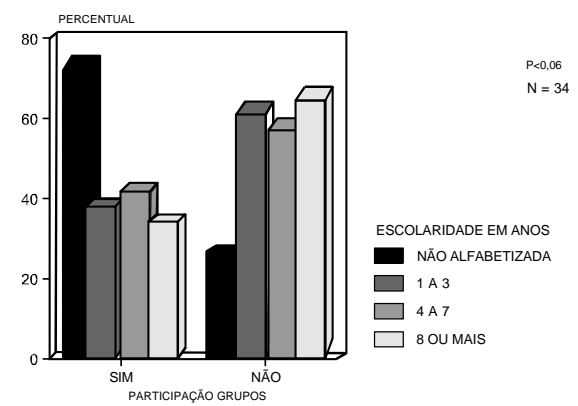

Fonte: Unidades Sanitárias UFPel-FAU, Pelotas, RS (Set, 1990).

FIGURA 2. Distribuição das Crianças Conforme Peso ao Nascer e Idade na Ocasião do Desmame

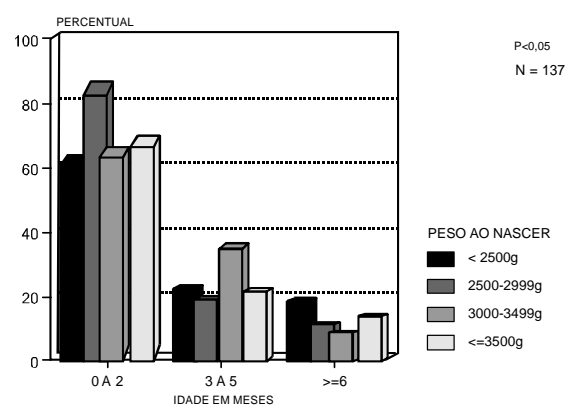

Fonte: Unidades Sanitárias UFPel-FAU, Pelotas, RS (Set, 1990).

FIGURA 3. Distribuição das Crianças Conforme a Época do Desmame, Introdução de Chá e Comida de Sal

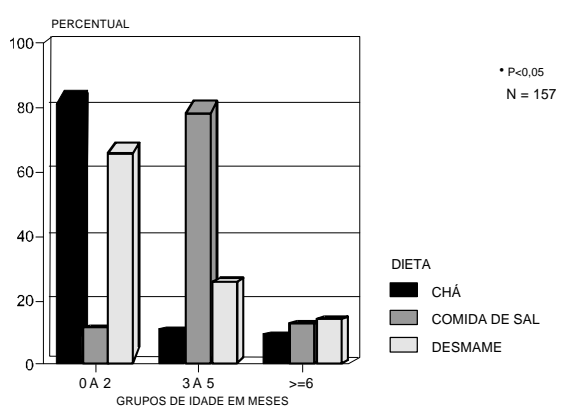

Fonte: Unidades Sanitárias UFPel-FAU, Pelotas, RS (Set, 1990). 
Não houve variação importante quanto à introdução de chás, comida de sal. peso ao nascer, época do desmame, renda familiar e escolaridade materna. Entretanto, estes resultados devem ser encarados com muita cautela, em virtude desta ser uma amostra de tamanho bastante reduzido.

\section{DISCUSSÃO}

Apesar da maioria das mulheres ter realizado pelo menos uma consulta de pré-natal e de $40 \%$ delas terem participado dos grupos de gestantes, $1 / 3$ das crianças já haviam sido desmamadas nos três primeiros meses de vida. Este achado está de acordo com a afirmação sobre a associação inversa entre utilização de alguns serviços de saúde e duração do aleitamento natural (Martins Filho, 1987; Sigulem \& Tudisco, 1980; Gomes, 1992).

A ausência de impacto da escolaridade materna e da participação nos grupos de gestantes deve ser vista com cautela, devido ao tamanho da amostra. Entretanto, esta mesma tendência já foi observada em outro estudo (Souza, 1991).

A época do desmame foi bastante precoce, independente da renda, escolaridade e peso ao nascer. Isto chama a atenção em virtude de as crianças pertencentes às famílias de baixo poder aquisitivo, principalmente as nascidas com baixo peso $(<2500 \mathrm{~g})$, apresentarem um maior coeficiente de morbi-mortalidade nos primeiros anos de vida (Victora et al., 1988; Xavier et al., 1991).

É bastante preocupante o elevado percentual de crianças que receberam chás durante os primeiros meses de vida. Considerando-se a elevada ocorrência de desmane até o segundo mês de vida, é possível sugerir que esta prática seja um elemento importante na determinação deste processo (Souza, 1991; Gomes, 1992). Além disso, não houve nenhum impacto, tanto das reuniões quanto do pré-natal, em retardar o uso de chás nos primeiros meses de vida.

$O$ resultado obtido para a mediana de amamentação de 4,27 meses é pouco superior ao observado em Pelotas, em 1982, apesar de, nesta última, ter-se verificado que as mães de menor poder aquisitivo amamentavam por menos tempo (Victora et al., 1988).
Os resultados obtidos mostram que existem importantes deficiências no trabalho de incentivo ao aleitamento nos locais estudados. Chama a atenção, ainda, o fato desta prática ser considerada prioridade em APS e destas unidades serem utilizadas como atividade prática por vários alunos dos cursos de graduação da área da saúde, os quais provavelmente irão reproduzir em sua vida profissional os padrões aprendidos durante sua formação.

\section{RECOMENDAÇÕES}

Apesar deste estudo ter sido realizado com uma amostra de tamanho bastante reduzido, de não ser representativo da população atendida por estas unidades sanitárias e de tratar de questões determinadas historicamente pela estrutura das sociedades, é possível acreditar que alguns aspectos específicos possam ser modificados pela ação dos profissionais de saúde.

Assim, a partir dos resultados obtidos, algumas medidas simples e de baixo custo poderiam ser adotadas, tais como:

- avaliar periodicamente os programas, incluindo as crianças que não frequentam o serviço rotineiramente, através de visita domiciliar;

- considerar o nível de escolaridade e renda familiar das mães ao elaborar o programa e promover discussões;

- priorizar e intensificar o acompanhamento das crianças, principalmente se de baixo peso;

- desestimular a introdução precoce de sucos e, principalmente, chás. 


\section{RESUMO}

NEUTZLING, M. B.; VIEIRA, M. F.; CÉSAR, J. A.; GIGANTE, D. P.; MARTINS, E. B. \& FACCHINI, L. A. Medindo o Impacto da Promoção do Aleitamento Materno em Serviços de Atenção Primária à Saúde em Pelotas, Rio Grande do Sul, Brasil. Cad. Saúde Públ., Rio de Janeiro, 9 (2): 149-154, abr/jun, 1993.

Através da aplicação de um questionário padronizado a todas as mães que procuraram os serviços de puericultura das unidades sanitárias (US) da Universidade Federal de Pelotas (UFPel), RS, em setembro de 1990, buscou-se conhecer o impacto dos grupos de gestantes na promoção do aleitamento materno. Os resultados obtidos para 347 crianças revelaram que quase metade delas pertencia a famílias com renda inferior a dois salários mínimos mensais; $1 / 4$ de suas mães apresentavam escolaridade inferior a quatro anos, sendo estas as que mais frequentaram os grupos. Apesar da maioria das mães ter feito pré-natal e pouco menos da metade ter participado dos grupos de gestantes, 1/3 das crianças estavam desmamadas aos três meses de idade e cerca de $80 \%$ delas haviam recebido chás nos primeiros meses. Os dados obtidos revelam um serviço com sérias deficiências em incentivar o aleitamento e em retardar a introdução de outros alimentos na dieta infantil. Espera-se, a partir dos dados obtidos, poder contribuir para a reestruturação dos programas existentes, bem como poder demonstrar que, a partir de recursos mínimos e de uma metodologia relativamente simples, é possível avaliar a qualidade dos serviços oferecidos à população.

Palavras-Chave: Aleitamento Materno; Saúde Infantil; Gravidez; Saúde Comunitária
BOLETIN AAPH (Boletin da Associação Americana de Saúde Pública), 1990. Boletin Sobre Alimentación y Nutrición Materna. Madre e Niños. Washington, DC: Centro de Documentação sobre Alimentação Infantil e Nutrição Materna; Associação Americana de Saúde Pública.

GRANT, J., 1992. Situação Mundial da Infância 1991. Brasília: Unicef.

GOMES, A. C. S., 1992. Avaliação do conhecimento das puérperas. Jornal de Pediatria, 68: 123-134.

JELLIFFE, D. B. \& JELLIFFE, E. P. F., 1978. Human Milk on the Modern World. New York: Oxford University Press.

KIRKWOOD, B. R., 1988. Essentials of M edical Statistics. Oxford and London: Blackwel Scientific Publications.

MANCIAX, M., 1982. Amamentação materna e doenças infecciosas nos países em desenvolvimento. Jornal de Pediatria, 53: 35-44.

MARTINS FILHO, J., 1987. Como e Porque Amamentar. $2^{\mathrm{a}}$ edição, São Paulo: Ed. Sarvier.

MONTEIRO, C. A., 1988. Saúde e Nutrição das Crianças de São Paulo. Diagnósticos, Contrastes Sociais e Tendências. São Paulo: Hucitec-Edusp.

SIGULEM, D. M. \& TUDISCO, E. S., 1980. Aleitamento natural em diferentes classes de renda no município de São Paulo. Archivos Latinoamericanos de Nutrición, 30: 401-416.

SOUZA, L. S. F; SOUZA, E. L. S.; BARRETTO, M. R. R.; RAMOS, R. T. T.; MACEDO, J. J. B.; SERRA, C. R. \& FERNANDES, C. E., 1991. Determinantes do êxito do aleitamento natural. Jornal de Pediatria, 67: 42-50.

VICTORA, C. G.; BARROS, F. C. \& VAUGHAN, J. P., 1988. Epidemiologia da Desigualdade. Um Estudo Longitudinal de 6000 Crianças Brasileiras. $2^{\mathrm{a}}$ edição, São Paulo: Hucitec.

XAVIER, C. C.; JORGE, S. M. \& GONÇALVES, A. L., 1991. Prevalência de aleitamento materno em recém-nascidos de baixo peso. Revista de Saúde Pública, 25: 381-387.

\section{REFERÊNCIAS BIBLIOGRÁFICAS}

BARROS, F. C. \& VICTORA, C. G., 1991. Epidemiologia da Saúde Infantil: um Manual para Diagnósticos Comunitários. Brasília: HucitecUnicef. 